\title{
Boundary Extraction in Natural Images Using Ultrametric Contour Maps
}

\author{
Pablo Arbeláez \\ CEREMADE, UMR CNRS 7534 Université Paris Dauphine, \\ Place du maréchal de Lattre de Tassigny, 75775 Paris cedex 16, France \\ arbelaez@ceremade. dauphine.fr
}

\begin{abstract}
This paper presents a low-level system for boundary extraction and segmentation of natural images and the evaluation of its performance. We study the problem in the framework of hierarchical classification, where the geometric structure of an image can be represented by an ultrametric contour map, the soft boundary image associated to a family of nested segmentations. We define generic ultrametric distances by integrating local contour cues along the regions boundaries and combining this information with region attributes. Then, we evaluate quantitatively our results with respect to ground-truth segmentation data, proving that our system outperforms significantly two widely used hierarchical segmentation techniques, as well as the state of the art in local edge detection.
\end{abstract}

\section{Introduction}

Hierarchical organization is one of the main characteristics of human segmentation. A human subject segments a natural image by identifying physical objects and marking their boundaries up to a certain level of detail. If we suppose that different subjects segmenting a single image perceive the same objects then, up to variations in the exact localization of boundaries, the intersection of their segments determines the finest level of detail considered. Figure 1 presents an example, with a natural image and six human segmentations from [9]. The segmentations are superimposed and the segments are represented by their mean color.

Segmentation can be thought as a process of grouping visual information, where the details are grouped into objects, objects into classes of objects, etc. Thus, starting from the composite segmentation, the perceptual organization of the image can be represented by a tree of regions, ordered by inclusion. The root of the tree is the entire scene, the leaves are the finest details and each region represents an object at a certain scale of observation.

In this paper, we study the segmentation problem in the framework of data classification, where the notions of tree

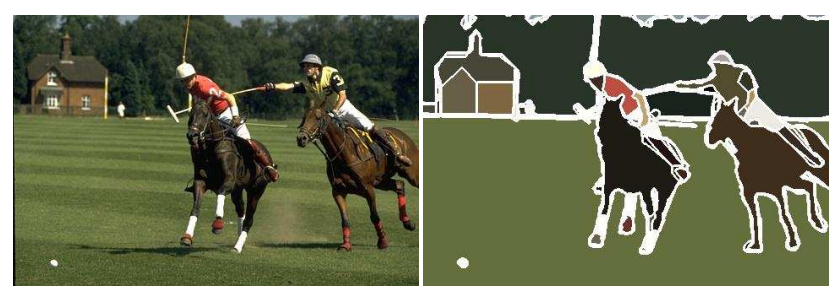

Figure 1. Natural image and composite human segmentation.

of regions and scale are formalized by the structure of indexed hierarchy or, equivalently, by the definition of an ultrametric distance on the image domain. We present a formulation of the problem in terms of contours that allows to represent an indexed hierarchy of regions as a soft boundary image called ultrametric contour map.

Since the early days of computer vision, the hierarchical structure of visual perception has motivated clustering techniques to segmentation [13], where connected regions of the image domain are classified according to an inter-region dissimilarity measure. We consider the traditional bottomup approach, also called agglomerative clustering, where the regions of an initial partition are iteratively merged, and we characterize those dissimilarities that are equivalent to ultrametric distances.

The key point of hierarchical classification is the definition of a specific distance for a particular application. We design ultrametrics for contour extraction by integrating and combining local edge evidence along the regions boundaries and complementing this information with intra-region attributes.

In order to design a generic segmentation system, all its degrees of freedom are expressed as parameters, whose tuning we interpret as the introduction of prior knowledge about the objects geometry. This information is provided by a database of human segmentations of natural images [9]. We optimize our system with respect to this groundtruth data and evaluate quantitatively our results using the Precision-Recall framework [8]. With this methodology, we prove that our system outperforms significantly two meth- 
ods of reference in segmentation by clustering, the variational approach of [7] and the hierarchical watersheds [12]. Furthermore, we show that the performance of our method is superior to state of the art local edge detectors [8], while providing a set of closed curves for any threshold.

\section{Definitions}

\subsection{Hierarchical Segmentation}

Let $\Omega \subset \mathbb{R}^{2}$ be the domain of definition of an image, $\mathcal{P}_{0}$ an initial partition of $\Omega$ and $\lambda \in \mathbb{R}$ a scale parameter. A hierarchical segmentation operator (HSO) is an application that assigns a partition $\mathcal{P}_{\lambda}$ to the couple $\left(\mathcal{P}_{0}, \lambda\right)$, such that the following properties are satisfied:

$$
\begin{gathered}
\mathcal{P}_{\lambda}=\mathcal{P}_{0}, \forall \lambda \leq 0 \\
\exists \lambda_{1} \in \mathbb{R}^{+}: \mathcal{P}_{\lambda}=\{\Omega\}, \forall \lambda \geq \lambda_{1} \\
\lambda \leq \lambda^{\prime} \Rightarrow \mathcal{P}_{\lambda} \sqsubseteq \mathcal{P}_{\lambda^{\prime}}
\end{gathered}
$$

Relations (1) and (2) determine the effective range of scales of the operator and indicate that the analysis can be restricted to the interval $\left[0, \lambda_{1}\right]$. The symbol $\sqsubseteq$ denotes the partial order of partitions : $\mathcal{P} \sqsubseteq \mathcal{P}^{\prime} \Leftrightarrow \forall a \in \mathcal{P}, \exists b \in$ $\mathcal{P}^{\prime}: a \subseteq b$. Thus, relation (3) sets that partitions at different scales are nested, imposing a hierarchical structure to the family $\mathcal{H}=\left\{R \subset \Omega \mid \exists \lambda: R \in \mathcal{P}_{\lambda}\right\}$.

By considering the scale where a region appears in $\mathcal{H}$, one can then define a stratification index, the real valued application $f$ given by :

$$
f(R)=\inf \left\{\lambda \in\left[0, \lambda_{1}\right] \mid R \in \mathcal{P}_{\lambda}\right\}, \forall R \in \mathcal{H}
$$

The couple $(\mathcal{H}, f)$ is called an indexed hierarchy of subsets of $\Omega$. It can be represented by a dendrogram, where the height of a region $R$ is given by $f(R)$. The construction of $(\mathcal{H}, f)$ is equivalent to the definition of a distance between two elements $x, y$ of $\mathcal{P}_{0}$ :

$$
\Upsilon(x, y)=\inf \{f(R) \mid x \in R \wedge y \in R \wedge R \in \mathcal{H}\} .
$$

The application $\Upsilon$ belongs to a special type of distances called ultrametrics that, in addition to the usual triangle inequality, satisfy the stronger relation :

$$
\Upsilon(x, y) \leq \max \{\Upsilon(x, z), \Upsilon(z, y))\} .
$$

\subsection{Ultrametric Contour Maps}

We now follow [10] for the definition of a segmentation in terms of contours in a continuous domain.

A segmentation $K$ of an image $u$ as a finite set of rectifiable Jordan curves, called the contours of $K$. The regions of $K$, noted $\left(R_{i}\right)_{i}$ are the connected components of $\Omega \backslash K$. Furthermore, we suppose that the contours meet

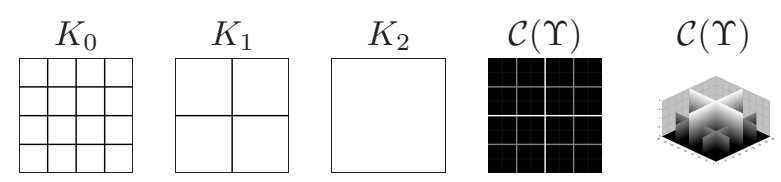

Figure 2. From left to right : Family of segmentations defined by a HSO, UCM and 3D view of UCM.

each other and $\partial \Omega$ only at their tips, that each contour separates two different regions and that each tip is common to at least three contours. The contour separating regions $R_{i}$ and $R_{j}$ is noted $\partial_{i j}$.

Hence, a segmentation can be equivalently expressed by its contours $K$ or by the partition $\mathcal{P}=\left\{\overline{R_{i}}\right\}_{i}$ of $\Omega$.

Rewriting properties (1) to (3) in terms of contours of the segmentations leads to the following characterization of a HSO :

$$
\begin{gathered}
K_{\lambda}=K_{0}, \forall \lambda \leq 0 \\
K_{\lambda}=\partial \Omega, \forall \lambda \geq \lambda_{1} \\
\lambda \leq \lambda^{\prime} \Rightarrow K_{\lambda} \supseteq K_{\lambda^{\prime}}
\end{gathered}
$$

Relation (7) determines the set of initial contours $K_{0}$ and (8) indicates that all inner contours vanish at finite scale. The hierarchical structure imposed by (9), called the principle of strong causality in [10], establishes that the localization of contours is preserved through the scales.

We can now define the model of contours that will be considered.

Let $\Upsilon$ be the ultrametric distance defined by a HSO. The ultrametric contour map (UCM) associated to $\Upsilon$ is the application $\mathcal{C}(\Upsilon): K_{0} \rightarrow\left[0, \lambda_{1}\right]$ given by:

$$
\mathcal{C}(\Upsilon)(\partial)=\inf \left\{\lambda \in\left[0, \lambda_{1}\right] \mid \partial \nsubseteq K_{\lambda}\right\}, \forall \partial \in K_{0} .
$$

The number $\mathcal{C}(\Upsilon)(\partial)$ is called the saliency of contour $\partial$. Note the duality with the regions, the saliency of $\partial$ being its scale of disappearance from the hierarchy of contours.

The ultrametric contour map is a representation of a HSO in a single real-valued image. Figure 2 presents a simple example of UCM. By definition, thresholding this soft boundary image at scale $\lambda$ provides a set of closed curves, the segmentation $K_{\lambda}$.

Nevertheless, note that if the initial partition $\mathcal{P}_{0}$ is fixed, all the UCM weight the elements of the same set $K_{0}$. Hence, the utility of such a representation is determined by the distance $\Upsilon$, whose value defines the saliency of each contour. Our objective will be to design ultrametrics such that the UCM model the boundaries of physical objects in natural images. 


\section{Ultramteric Dissimilarities}

\subsection{Region Merging}

The ascending construction of a family of nested partitions is one of the earliest approaches to segmentation $[10,4]$. These region merging techniques are principally determined by two elements : the choice of an initial partition $\mathcal{P}_{0}$ and the definition of a dissimilarity measure $\delta$ between couples of adjacent regions.

Such a merging process can be implemented efficiently by using a region adjacency graph (RAG) [14, 6]. A RAG is an undirected graph where the nodes represent connected regions of the image domain. The links encode the adjacency relation and are weighted by the dissimilarity. The RAG is initialized with $\mathcal{P}_{0}$ and the family of partitions is obtained by removing the links for increasing values of the dissimilarity and merging the corresponding nodes.

In the huge literature on the subject, the initial partition is typically an over-segmentation in very homogeneous regions, e.g. the partition in pixels, in connected components or the result of a pre-processing. The dissimilarity measure is defined from the image data. One can for instance consider a piecewise regular approximation of the initial image and measure the dissimilarity by comparing the approximations of adjacent regions.

\subsection{Defining the Distance}

Starting from a family of nested partitions constructed by a region merging process, one can always define an ultrametric distance by considering as stratification index an increasing function of the merging order. However, in order to define a meaningful notion of scale, the distance between any two points in adjacent regions should coincide with the inter-region dissimilarity $\delta$ :

$$
\Upsilon(x, y)=\delta\left(R_{i}, R_{j}\right), \forall x \in R_{i}, \forall y \in R_{j} .
$$

This property is satisfied by setting the value of the dissimilarity at the creation of a region as its stratification index. However, for an arbitrary dissimilarity, this choice can lead to the existence of two regions $\left(R, R^{\prime}\right) \in \mathcal{H}^{2}$ such that $R \subset R^{\prime}$ but $f(R)>f\left(R^{\prime}\right)$. In terms of contours, this situation implies the violation of strong causality (9).

Hence, we call $\delta$ an ultrametric dissimilarity if the couple $(\mathcal{H}, f)$ is an indexed hierarchy, where $f$ is defined by :

$$
f\left(R_{i} \cup R_{j}\right)=\delta\left(R_{i}, R_{j}\right),
$$

for all couples of connected regions $\left(R_{i}, R_{j}\right) \in \mathcal{H}^{2}$.

One can then prove that a dissimilarity $\delta$ is ultrametric if and only if

$$
\delta\left(R_{i}, R_{j}\right) \leq \delta\left(R_{i} \cup R_{j}, R\right),
$$

where $\left(R_{i}, R_{j}\right)$ is a couple of regions that minimizes $\delta$ and $R$ is a region connected to $R_{i} \cup R_{j}$ and belonging to the partition obtained after the merging of $\left(R_{i}, R_{j}\right)$.

In order to stress the fact that ultrametric dissimilarities define distances in $\mathcal{P}_{0}$, they are noted $\Upsilon$ in the sequel.

Working with ultrametric dissimilarities, as those defined in the next section, is important for our application because it guarantees that the saliency of each contour in the UCM is exactly the value of the dissimilarity between the two regions it separates.

\section{Ultrametrics for Boundary Extraction}

We now turn to the problem of defining specific distances for the extraction of boundaries in natural images. For this purpose, our strategy consists in measuring local contour evidence along the common boundary of regions, and then complementing this information with internal region attributes. In order to design a generic low-level system, all the degrees of freedom are expressed as parameters.

\subsection{A Contrast Ultrametric}

We first define a distance that uses the color information of the initial image for measuring the contrast between regions. For this purpose, we represent a color image $u$ with the $L^{*} a b$ standard [15], an approximation of the perceptually uniform color space. The distance between two colors in $L^{*} a b$ is usually measured by the Pythagorean formula; however, in order to tolerate variations in the relative weight of lightness and chrominance, we propose the following distance $d^{*}$ between two colors $k=(l, a, b)$ and $k^{\prime}=\left(l^{\prime}, a^{\prime}, b^{\prime}\right)$ :

$$
d^{*}\left(k, k^{\prime}\right)=\sqrt{\left(l-l^{\prime}\right)^{2}+\xi\left(a-a^{\prime}\right)^{2}+\xi\left(b-b^{\prime}\right)^{2}} .
$$

With this color representation, monochromatic images correspond to the particular case $\xi=0$ and the usual $L^{*} a b$ space to $\xi=1$.

In order to determine the presence of a boundary, we define a local contrast measure at a point $x \in \Omega$ by the formula:

$$
\tau(u, x)=\sup \left\{d^{*}\left(u(y), u\left(y^{\prime}\right)\right) \mid \forall y, y^{\prime} \in B_{r}(x)\right\},
$$

where $B_{r}(x)$ denotes a Euclidian ball of radius $r$ centered at $x$.

Our first distance is given by the mean local contrast along the common boundary of regions:

$$
\Upsilon_{c}\left(R_{i}, R_{j}\right)=\frac{\Sigma_{c}\left(\partial_{i j}\right)}{L\left(\partial_{i j}\right)},
$$

where $L(\partial)$ denotes the length of the contour $\partial$ and $\Sigma_{c}(\partial)$ is given by :

$$
\Sigma_{c}(\partial)=\int_{\partial} \tau(u, x(s)) d s
$$


The interest of distance $\Upsilon_{c}$ is that, being defined on the initial image $u$, the set $K_{0}$ of initial contours coincides with the discontinuities of $u$. Thus, the associated UCM preserve the localization of the original boundaries.

\subsection{A Boundary Ultrametric}

The distance $\Upsilon_{c}$ expresses a notion of homogeneity of objects based only on color uniformity. In order to consider a larger class of contour cues, we use as input to our system the result of a local edge detector, noted $g$. In the examples presented, we used the detector of [8], that measures and combines optimally local cues as brightness, color and texture.

As in the previous paragraph, we consider a mean boundary gradient distance:

$$
\Upsilon_{g}\left(R_{i}, R_{j}\right)=\frac{\Sigma_{g}\left(\partial_{i j}\right)}{L\left(\partial_{i j}\right)},
$$

where

$$
\Sigma_{g}(\partial)=\int_{\partial} g(x(s)) d s .
$$

Then, the weighted sum of mean boundary gradient and mean contrast of the initial image defines our final boundary ultrametric:

$$
\Upsilon_{B}\left(R_{i}, R_{j}\right)=\Upsilon_{c}\left(R_{i}, R_{j}\right)+\alpha_{1} \cdot \Upsilon_{g}\left(R_{i}, R_{j}\right),
$$

where $\alpha_{1} \geq 0$.

\subsection{Considering Region Attributes}

In order to take into account the internal information of regions in the distance, we measure an attribute on each region, a function $\mathcal{A}: \mathbb{P}(\Omega) \rightarrow \mathbb{R}^{+}$, increasing with respect to the inclusion order : $R \subset R^{\prime} \Leftrightarrow \mathcal{A}(R)<\mathcal{A}\left(R^{\prime}\right)$.

Thus, starting from (20), we define the following generic notion of scale for boundary extraction:

$$
\Upsilon_{S}\left(R_{i}, R_{j}\right)=\Upsilon_{B}\left(R_{i}, R_{j}\right) \cdot \min \left\{\mathcal{A}\left(R_{i}\right), \mathcal{A}\left(R_{j}\right)\right\}^{\alpha_{2}},
$$

where $\alpha_{2} \geq 0$. Note that, since the term $\Upsilon_{c}$ never vanishes, the UCM associated to $\Upsilon_{B}$ and $\Upsilon_{S}$ preserve the localization of the contours of the initial image.

In the experiments presented in this paper, we used as attribute a linear combination of area and total quadratic error:

$$
\mathcal{A}(R)=\int_{R} d x+\alpha_{3} \cdot \int_{R} d^{*}(u(x), M(R))^{2} d x
$$

where $\alpha_{3} \geq 0$ and $M(R)$ denotes the mean color of $u$ on region $R$.

We considered two methods for introducing the internal information in the distance. The first one is to use directly the UCM associated to $\Upsilon_{S}$. The second one consists in pruning the tree of $\Upsilon_{B}$ for increasing values of $\Upsilon_{S}$.

\subsection{Initial Set of Contours}

We have discussed so far the definition of distances meaningful for our application. We now address the issue of choosing an appropriated set of initial contours $K_{0}$. For this purpose, in absence of prior knowledge about the image content, we do the methodological choice of preserving the inclusion relation between the discontinuities of the original image and $K_{0}$. This amounts to supposing that a connected region where the image is constant cannot belong to two different objects. This property is satisfied by the presegmentation method we now describe.

The objective of pre-segmentation is to decompose the image in local entities, more coherent and less numerous than pixels, while preserving its geometric structure. For this purpose, we exploit the fact that ultrametric dissimilarities define pseudo-metrics on the image domain. Precisely, we produce an over-segmentation by considering the extrema of the $L^{*}$ channel in the original image and associating each point of $\Omega$ to the closest extremum in the sense of $\Upsilon_{S}$. This construction defines a Voronoi tessellation of the domain; a pre-segmented image is then obtained by assigning a constant color to each region.

Thus, the method produces a reconstruction of the original image from the information of its lightness extrema, that preserves the localization of the original contours. Depending on the image, the pre-segmentation can be iterated a few times without affecting the main geometric structures. Its degrees of freedom are treated as additional parameters of our system.

\subsection{Examples}

Figure 3 illustrates the influence on the output of our system of the different types of information considered in the proposed notion of scale. Lines 2 to 5 present, from left to right, the UCM, the segmentation given by the optimal scale of the UCM with respect to human-marked boundaries (see Section 5.1) and the corresponding reconstruction, where the segments are represented by their mean color. The distance $\Upsilon_{c}$ of Line 2 measures only the contrast of the original image. As a consequence, textured regions as the dress are a source of noise in the UCM. The saliency of these regions is reduced in the boundary ultrametric $\Upsilon_{B}$ (Line 3 ) by choosing a local detector that measures texture homogeneity. The second example shows the utility of considering internal region attributes (Line 5) as a complement to plain boundary information (Line 4). On the one hand, it eliminates small imperfections of the image. On the other hand, it allows to control the saliency of structures like the dark dots, whose size triggers the response of the local detector, but whose importance may depend on the application. 

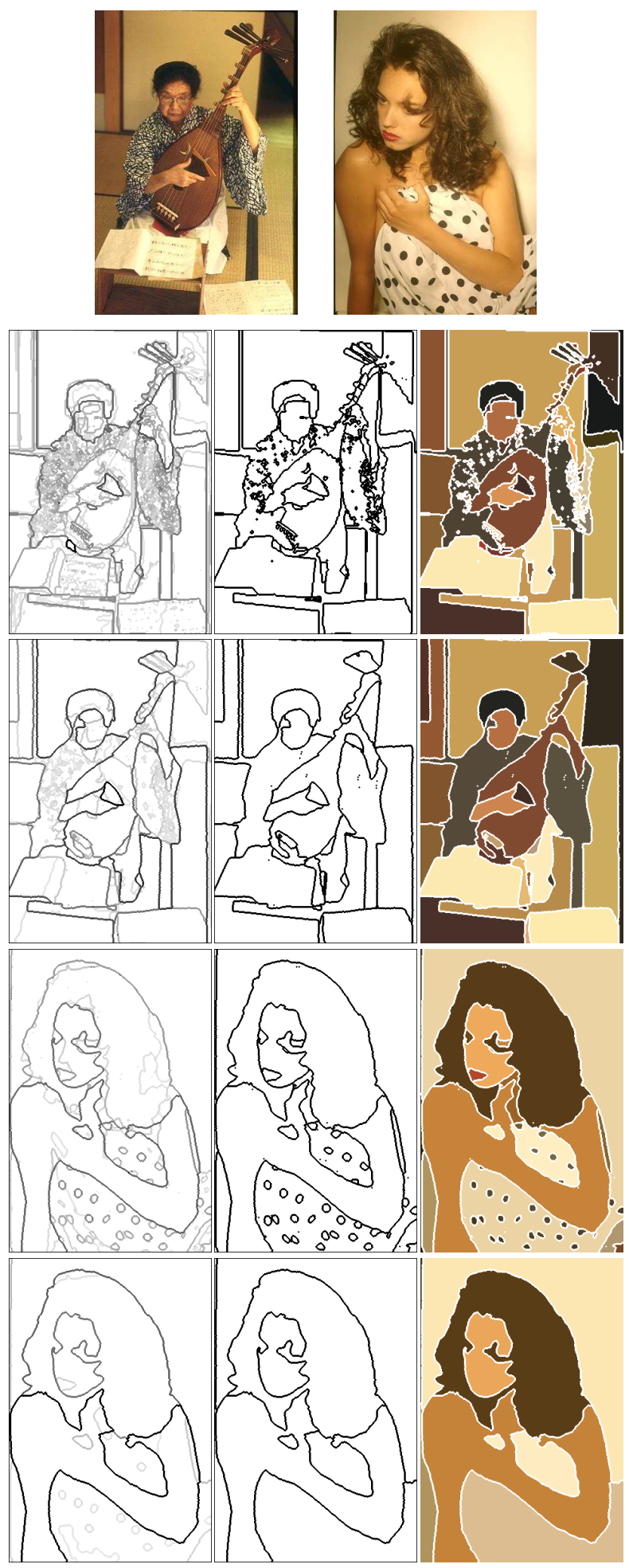

Figure 3. Examples. Line 1: original images. Lines 2 to 5 . From left to right : UCM, segmentation and reconstruction. From top to bottom: $\mathcal{C}\left(\Upsilon_{c}\right), \mathcal{C}\left(\Upsilon_{B}\right), \mathcal{C}\left(\Upsilon_{B}\right)$ and $\mathcal{C}\left(\Upsilon_{S}\right)$.

\section{Evaluation of Results}

\subsection{Methodology}

Our system combines several types of low-level image information into a single notion of scale, the saliency of boundaries in the UCM. We interpret the tuning of its parameters as the introduction of prior information about the geometric structure of the image. In order to measure quantitatively the pertinence of our approach and compare it to other methods, the system was optimized using the Berkeley Segmentation Dataset and Benchmark (BSDB) [1] .

In this study, the ground-truth data is provided by a large database of natural images, manually segmented by human subjects [9]. A methodology for evaluating the performance of boundary detection techniques with this database was developed in [8]. It is based in the comparison of machinedetected boundaries with respect to human-marked boundaries using the Precision-Recall framework, a standard information retrieval technique. Precisely, two quality measures are considered, Precision $(\mathrm{P})$, defined as the fraction of detections that are true boundaries and Recall (R), given by the fraction of true boundaries that are detected. Thus, Precision quantifies the amount of noise in the output of the detector, while Recall quantifies the amount of ground-truth detected. Measuring these descriptors over a set of images for different thresholds of the detector provides a parametric Precision-Recall curve. The two quantities are then combined in a single quality measure, the $F$-measure, defined as their harmonic mean:

$$
F(P, R)=\frac{2 P R}{P+R} .
$$

Finally, the maximal F-measure on the curve is used as a summary statistic for the quality of the detector on the set of images.

The current public version of the BSDB is divided in two independent sets of images. A training set of 200 images and a test set of 100 images. In order to ensure the integrity of the evaluation, only the images and segmentations from the training set can be accessed during the optimization phase. The algorithm is then benchmarked by using the optimal training parameters "blindly" on the test set.

\subsection{Tested Methods}

The BSDB is a standard experimental protocol that allows the comparison of different boundary detection techniques by quantifying their performance with respect to human vision. Furthermore, since the quality of a detector is judged exclusively on the accuracy of the contours it produces, the methodology permits a direct comparison between local edge detection and region-based segmentation approaches. Although its use in the segmentation community is nowadays widespread, only the former have been 
evaluated to our knowledge (see [8]). Estrada and Jepson [3] compared recently four region-based segmentation methods within the same framework. However, it should be noted that their study uses a different experimental setup and considers only gray-level images.

Our evaluation work was divided in two parts. First, optimizing and benchmarking our system in order to compare it to already benchmarked local edge detectors. Second, we benchmarked two methods of reference in hierarchical segmentation and compared their results with ours. The optimization phase was carried out using a steepest ascent in the F-measure. In all the experiments, we used the implementations made available by the authors. We now describe briefly the tested algorithms.

\subsubsection{Local Edge Detectors}

The state of the art in local boundary detection techniques is the system proposed in $[8,1]$. The gradient paradigm implemented by Martin et al. relies on the measure, at each pixel, of local discontinuities in feature channels like brightness, color and texture, over a range of scales and orientations. Local cues are combined and optimized with respect to the BSDB. Then, using the Precision-Recall framework, the authors show that their detector, noted in the sequel MFM, outperforms all other local edge detection methods.

We used the MFM as a reference of local detection methods and as an input to our system. Additionally, the Canny detector, based on an estimation of the gradient magnitude of brightness and non-maxima suppression, provided the baseline in classical local approaches. For both methods, we used the codes distributed with the BSDB with their default optimized parameters.

\subsubsection{Hierarchical Watersheds}

As a reference of a hierarchical segmentation operator based only the information of a gradient image, we used the work of Najman and Schmitt [12,2], who study the ultrametric contour map associated to the watershed segmentation. To our knowledge, these authors were the first to consider explicitly the soft contour image associated to a family of nested segmentations for boundary detection purposes.

This method is based on the construction of the watershed by a flooding process. A gradient image, seen as a topographic surface, is pierced at its minima and progressively immersed in water. The water fills the catchment basins of the minima and forms lakes. When two lakes meet, the level of the water (the height of the saddle point) determines the saliency of the corresponding watershed arc. Thus, the notion of scale of this technique is an estimation of the contrast between two regions by the minimal value of the gradient on their common boundary.
A natural choice for the topographic surface seems to be the MFM detector. However, following the Canny approach, the MFM considers only the maxima in the gradient direction. As a consequence, the final output of the MFM has often a single regional minimum and, in the presence of a unique lake, the watershed is empty. Thus, we used as topographic surface the MFM detector before non-maxima suppression. This choice has the interest of providing a watershed for color images, with regular contours and robust to noise and texture.

\subsubsection{Variational Approach}

The formulation of the segmentation problem in the variational framework is one of the most popular approaches of the recent years. In order to compare our results to a HSO based exclusively on the information of the original image, we used the work of Koepfler et al. [7, 10, 5], who propose a multi-scale approach for the minimization of the piecewise constant version of the Mumford and Shah functional [11].

This study considers a region merging process, where the criterion to merge two regions is to decrease the value of the global energy:

$$
E(K)=\int_{\Omega \backslash K}\|m-u\|^{2} d x+\lambda L(K),
$$

where $m$ denotes the piecewise constant approximation of the initial image $u$ by the mean color in each region. Hence, with this approach, the objects in the image are modelled as regions of homogenous color with short boundaries. The dissimilarity governing this merging process is given by:

$$
\delta_{M S}\left(R_{i}, R_{j}\right)=\frac{\left|R_{i}\right| \cdot\left|R_{j}\right|}{\left|R_{i}\right|+\left|R_{j}\right|} \cdot \frac{\left\|M\left(R_{i}\right)-M\left(R_{j}\right)\right\|^{2}}{L\left(\partial_{i j}\right)},
$$

where $|R|$ denotes the area of region $R$. Thus, $\delta_{M S}$ is the squared absolute difference of the mean color in the regions, combined with two other factors. The first one is proportional to the harmonic mean of the areas and eliminates small regions from the segmentation. The second one is the inverse of the boundary length. This information controls the regularity of the result, since, if the other factors of $\delta_{M S}$ are identical, the couple of regions with longest common boundary is merged first. However, note that the dissimilarity $\delta_{M S}$ is not ultrametric. In order to restore strong causality, we constructed the UCM of this method by superimposing the results for increasing values of the parameter $\lambda$ in (24).

In order to make the comparison with our system fair, we used the $L^{*} a b$ space with the weight $\xi$ in (14) tuned for this algorithm. Additionally, we optimized as initial image the result of the pre-segmentation method described in Section 4.4. 

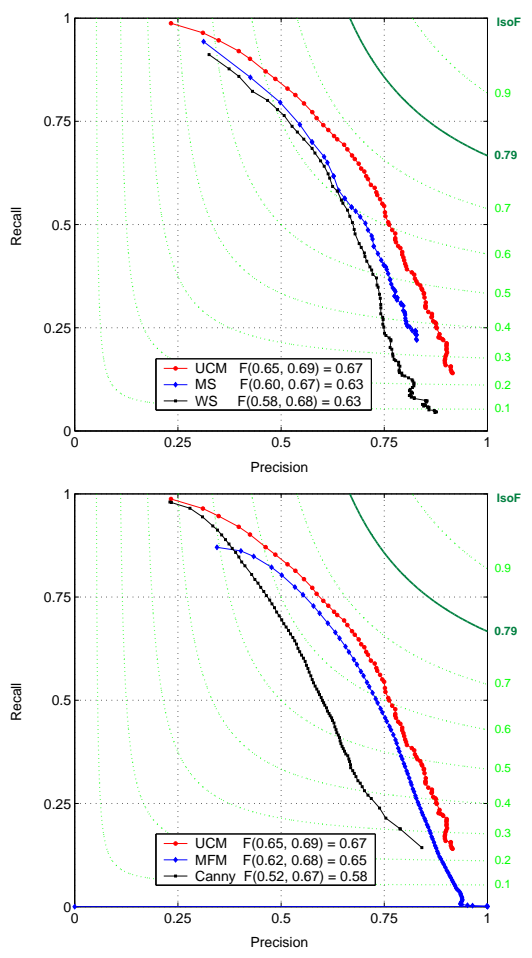

Figure 4. Evaluation results. Up: our UCM compared to other clustering techniques. Down: our UCM compared to local edge detectors

\subsection{Results}

The results of the evaluation on the test set are presented in Figure 4. The dotted (green) lines represent the isolevel lines of the function $F(P, R)$ defined by (23), with values on the right. They can be used to situate the PrecisionRecall curves, since the quality of a detector is judged by considering the image of its curve by $F$. Thus, the point with maximal F-measure can be interpreted as the highest point of the curve on the surface $z=F(P, R)$, the objective being the point $F(1,1)=1$. The isolevel line $F(P, R)=0.79$ corresponds to the human consistency on this set of images, obtained by comparing the human segmentations among them. This line represents the reference with respect to which the performance of machines for the task of boundary extraction is measured.

It can be observed that the Precision-Recall curve of our system (UCM) dominates significantly all the others. Hence, for a given amount of noise tolerated (fixed Precision), our UCM detect a greater fraction of true boundaries (higher Recall). Conversely, for a required amount of ground-truth detected (fixed Recall), our results are those with less noise (higher Precision).

The top panel shows the curves corresponding to the three HSOs benchmarked. Although the scores of the variational approach (MS) and the hierarchical watersheds (WS) are very close, the curve of the former dominates that of the latter. However, both methods are significantly outperformed by our system. The hierarchical watershed exploits the information of a gradient image, while the variational approach considers only the original image. The superiority of our UCM comes from the generic combination of both types of information.

The bottom panel compares our UCM with two local detectors already benchmarked on the BSDB: the classical Canny method and the top-ranked MFM. As before, our curve clearly dominates the two others. Nevertheless, more than the score, the main improvement of our system with respect to local detectors is to provide for any scale a set of closed curves, the contours of a segmentation.

Figures 5 and 6 present some qualitative results, all obtained with the optimal training parameters of each algorithm. In order to make the results completely automatic, the segmentation shown corresponds to the global optimal scale on the train set. Note that although our system was optimized using only boundary information, the segments obtained coincide often with physical objects or their connected parts.

Figure 6 compares the four tested methods on an example. It can be observed that our model of boundaries provides the best trade-off between accuracy and noise, allowing the extraction of the main geometric structures in the images. The improvement of our UCM with respect to the variational approach is in part due to the explicit treatment of texture of the local detector. The watersheds present the more regular contours among the tested methods and display a typical mosaic effect, where the lakes of the topographic surface are discernable in the contour map. Finally, the MFM results are shown to illustrate the essential difference in initial orientation between local and region-based approaches. The level of representation is the point for the former and the region for the latter. As a consequence, local detectors require in many applications a contour completion post-processing in order to integrate their information into closed curves.

\section{Conclusions}

This paper presented the design and evaluation of a novel boundary extraction system. Its main characteristic is to rely on the formulation of the problem in the framework of hierarchical classification, which allows to address regionbased segmentation and edge detection as a single task.

We defined generic ultrametric distances for this specific application and showed the interest of the approach followed by evaluating our results with a standard methodology. Additionally, we evaluated with the same protocol two methods of reference in hierarchical segmentation.

Although the introduction of semantic information seems necessary in order to fill the still large gap with hu- 


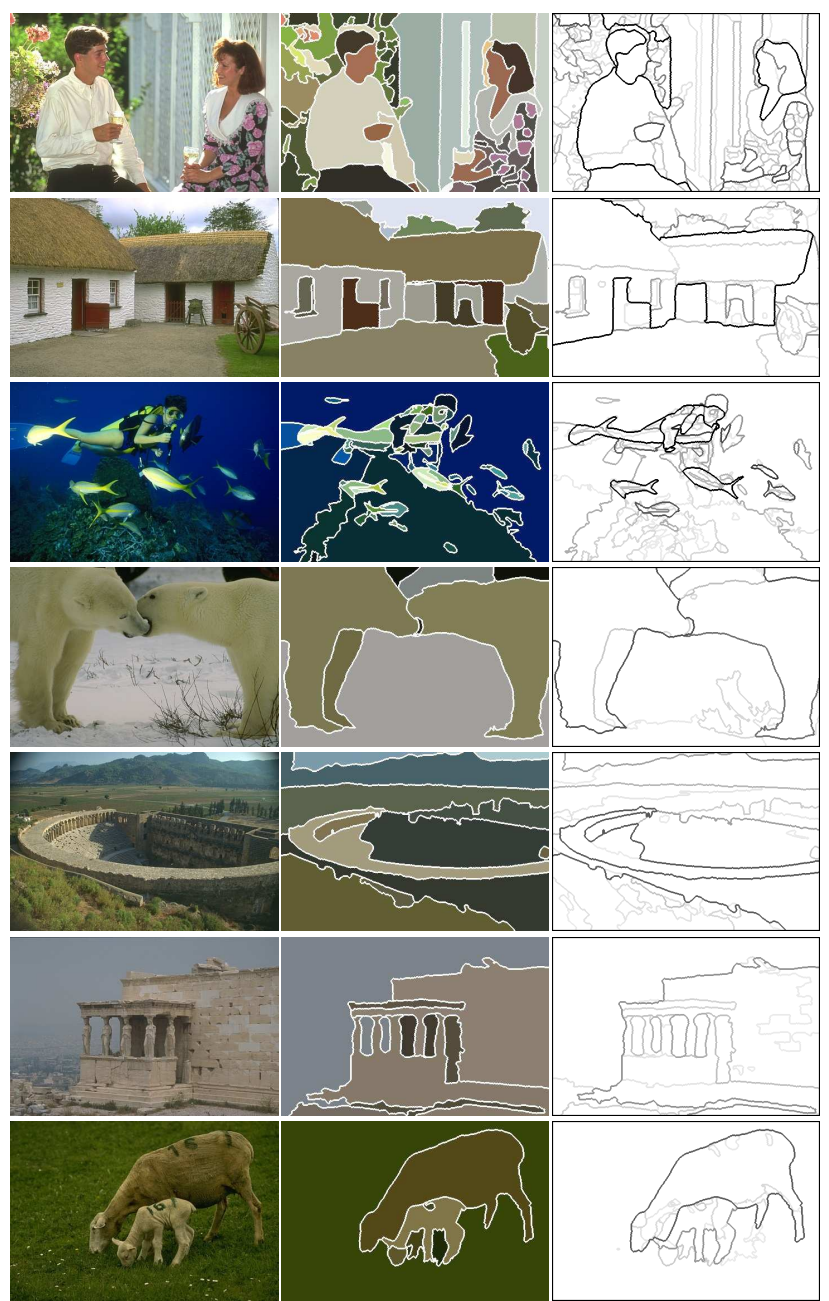

Figure 5. Results of our system. From left to right : Original images, automatic segmentation and UCM.

man performance for this task, we are confident that higherlevel approaches can benefit from our low-level representation of the image boundaries.

\section{References}

[1] The Berkeley Segmentation Dataset and Benchmark. www.cs.berkeley.edu/projects/vision/grouping/segbench/. 5, 6

[2] M. Couprie. Library of operators in image processing PINK. http://www.esiee.fr/ coupriem. 6

[3] F. Estrada and A. Jepson. Quantitative evaluation of a novel image segmentation algorithm. In Proc. CVPR'05, pages II: 1132-1139, 2005. 6

[4] D. Forsyth and J. Ponce. Computer Vision: A Modern Approach. Prentice Hall, 2003. 3

[5] J. Froment and L. Moisan. Image processing software megawave. http://www.cmla.enscachan.fr/Cmla/Megawave/. 6

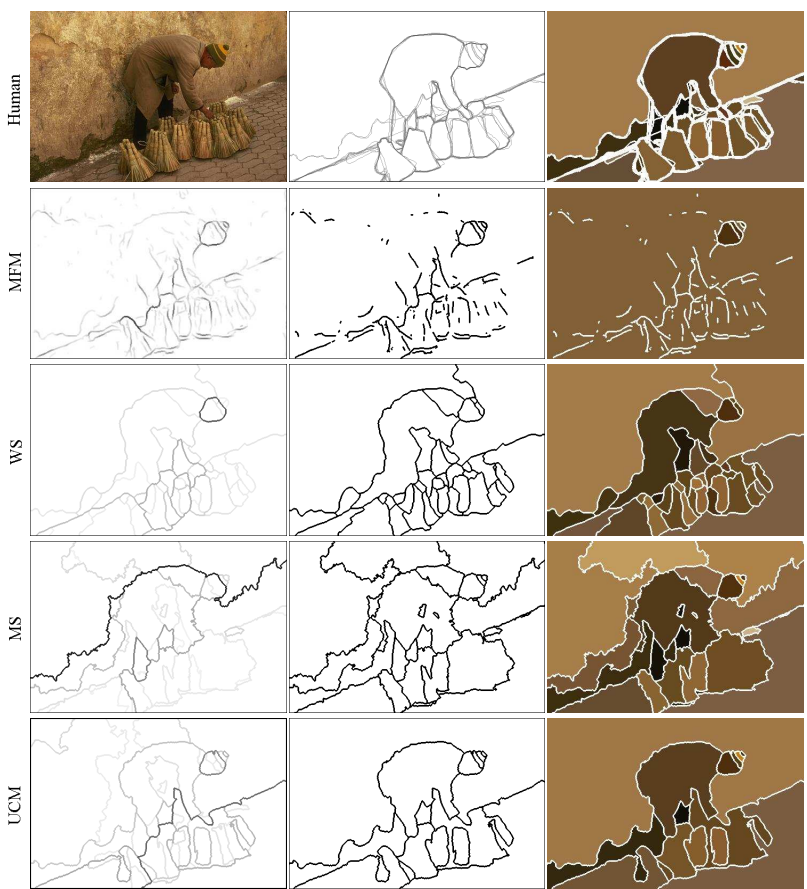

Figure 6. Comparison of the four tested methods (see text).

[6] L. Garrido, P. Salembier, and D. Garcia. Extensive operators in partition lattices for image sequence analysis. IEEE Trans. on Signal Processing, 66(2):157-180, Apr. 1998. 3

[7] G. Koepfler, C. Lopez, and J. Morel. A multiscale algorithm for image segmentation by variational method. SIAM Journal on Numerical Analysis, 31(1):282-299, 1994. 2, 6

[8] D. Martin, C. Fowlkes, and J. Malik. Learning to detect natural image boundaries using local brightness, color and texture cues. IEEE Trans. on PAMI, 26(5):530-549, 2004. 1, 2, $4,5,6$

[9] D. Martin, C. Fowlkes, D. Tal, and J. Malik. A database of human segmented natural images and its application. In Proc. ICCV'01, volume II, pages 416-423, 2001. 1, 5

[10] J. Morel and S. Solimini. Variational Methods in Image Segmentation. Birkhauser, 1995. 2, 3, 6

[11] D. Mumford and J. Shah. Optimal approximations by piecewise smooth functions and variational problems. CPAM, 42(5):577-684, 1989. 6

[12] L. Najman and M. Schmitt. Geodesic saliency of watershed contours and hierarchical segmentation. IEEE Trans. on PAMI, 18(12):1163-1173, 1996. 2, 6

[13] R. Ohlander, K. Price, and R. Reddy. Picture segmentation by a recursive region splitting method. Computer Graphics Image Processing, 8:313-333, 1978. 1

[14] T. Vlachos and A. Constantinides. Graph-theoretical approach to colour picture segmentation and contour classification. In IEE Proc. Vision, Image and Sig. Proc., volume 140, pages 36-45, Feb. 1993. 3

[15] G. Wyszecki and W. Stiles. Color Science: Concepts and Methods, Quantitative Data and Formulas. J. Wiley and Sons, 1982. 3 